\title{
The ubiquitin-conjugating enzyme E2-EPF is overexpressed in cervical cancer and associates with tumor growth
}

\author{
JING LIANG $^{1}$, HIROTAKA NISHI ${ }^{2}$, MEI-LU BIAN ${ }^{1}$, CHINATSU HIGUMA $^{2}$, \\ TORU SASAKI ${ }^{2},{\text { HIROE } \text { ITO }^{2} \text { and KEIICHI ISAKA }}^{2}$ \\ ${ }^{1}$ Department of Obstetrics and Gynecology, China-Japan Friendship Hospital, Beijing, P.R. China; \\ ${ }^{2}$ Department of Obstetrics and Gynecology, Tokyo Medical University, Tokyo, Japan
}

Received May 16, 2012; Accepted July 11, 2012

DOI: $10.3892 /$ or.2012.1949

\begin{abstract}
We found that the ubiquitin-conjugating enzyme E2-EPF mRNA is highly expressed in cervical squamous cancer relative to normal tissues and its expression levels positively correlate with clinical stage. Reduction of E2-EPF protein levels by $>80 \%$ using shRNA decreases the expression levels of HIF-1 $\alpha$, and the proliferation, invasion and tumorigenicity of $\mathrm{SiHa}$, a cervical squamous cancer cell line. E2-EPF knockdown also increases the chemosensitivity to topoisomerase I inhibitor (topotecan) and II (etoposide and doxorubicin). Our results suggest that E2-EPF is associated with the growth and aggressivity of cervical tumor cells. Targeting the E2-EPF pathway may have potential clinical applications for the treatment of cervical cancer.
\end{abstract}

\section{Introduction}

The ubiquitin-proteasome pathway is involved in tumorigenesis (1). E2-EPF is a 24-kDa protein that is a member of the E2 family of ubiquitin-conjugating enzymes (2), which, together with an E1 ubiquitin-activating enzyme and an E3 ubiquitin ligase, catalyze the addition of ubiquitin to substrate proteins (3). Multiple rounds of ubiquitinylation result in substrate polyubiquitinylation that can target proteins for proteasome-dependent destruction. E2-EPF was discovered in 1992 and was highly expressed in human cancer tissues compared with normal tissues $(4,5)$. Largescale meta-analysis of cancer microarray data identifies that E2-EPF is one of commonly activated genes in multiple cancer (6). Though discovered in 1992, candidate substrates and cognate E3 ligases for E2-EPF were unclear until Jung et al demonstrated

Correspondence to: Dr Hirotaka Nishi, Department of Obstetrics and Gynecology, Tokyo Medical University, 6-7-1 Nishishinjuku, Shinjuku-ku, Tokyo 160-0023, Japan

E-mail: nishih@tokyo-med.ac.jp

Professor Mei-Lu Bian, Department of Obstetrics and Gynecology, China-Japan Friendship Hospital, Beijing, P.R. China

E-mail: bianmeilu@hotmail.com

Key words: E2-EPF, HIF-1 $\alpha$, cervical cancer, RNAi, topoisomerase that the stability of a von Hippel-Lindau (VHL) tumorsuppressor gene product is dependent on E2-EPF levels in 2006 (7). As we know, human solid tumors contain hypoxic regions that have considerably lower oxygen tension than normal tissues. Hypoxia offers resistance to radiotherapy and anticancer chemotherapy, as well as predispose to increased tumor metastases. Furthermore, hypoxia induces hypoxia inducible factor (HIF)-1, which in turn increases tumor angiogenesis (8). The von Hippel-Lindau tumor suppressor, pVHL, forms part of an E3 ubiquitin ligase complex that targets specific substrates for degradation, including HIF-1 $\alpha$ (9). Their study suggested a role for E2-EPF in the stabilization of HIF-1 $\alpha$ by specifically targeting pVHL for degradation under normoxic conditions. E2-EPF expression level correlates inversely with pVHL level in most tumor cell lines. In vitro and in vivo, forced expression of E2-EPF boosts tumor-cell proliferation, invasion and metastasis through effects on the pVHL-HIF pathway (7).

Thereafter, increased expression of E2-EPF expression in multiple cancers including breast cancer (10) and esophageal squamous cell carcinoma (11) was reported and their roles in tumor cell growth and metastasis were explored. Cellular factors that regulate the expression of E2-EPF gene were also revealed. Growth factors and serum induce expression of Egr-1 and SRF, respectively. Egr-1 and SRF can bind the promotor region of E2-EPF, therefore increase the HIF-1 $\alpha$ protein level under non-hypoxic conditions through the Egr-1/SRF- E2-EPF-VHL pathway (12).

Here, we report the expression profile for E2-EPF in cervical tumor and normal tissue specimens and address that it has an essential role in cancer cell proliferation, invasion, tumorigenicity and chemosensitivity to topoisomerase inhibitors.

\section{Materials and methods}

Experimental samples. Samples from cervical cancer and normal cervical tissues were obtained with informed consent form patients undergoing surgery or biopsy in Tokyo Medical University Hospital. The protocols used here have been approved by the ethics committees of the respective institutions where their study was carried out and conform to the provisions of the Declaration of Helsinki in 1995 (as revised in Edinburgh 2000). Totally, 13 normal cervical tissues, and 75 cervical carcinoma tissues were analysed. The clinical stage of tumor tissues was 
classified by the FIGO 2009 standard. Tumor histological grades, as well as the status of chemotherapy were recorded. Sample tissues were minced into small pieces with scissors; washed in $0.9 \%$ sterile saline to avoid contamination of red blood cells, snap-frozen and stored at $-80^{\circ} \mathrm{C}$ until used.

Total RNA isolation and real-time PCR analysis. Total RNA was isolated using Isogen reagent (Nippon Gene, Tokyo, Japan) and was reverse transcribed into cDNA with High Capacity cDNA Reverse Transcription Kit (Applied Biosystems, USA). Real-time PCR was carried out for quantitative estimation with TaqMan Gene Expression Master Mix (Applied Biosystems). E2-EPF was amplified with the following primer pair: forward: 5'-CGACCTCCAGGTCACCAT-3'; reverse: 5'-GGAACAGACCTCCAGCATATGG-3' and with a probe 5'-CCCCTCAGGGCCCTC-3'. The reaction was cycled in the StepOne Plus real-time PCR system (Applied Biosystems) with the following parameters: denaturation for 1 cycle at $95^{\circ} \mathrm{C}$ for $10 \mathrm{~min}, 40$ cycles of $95^{\circ} \mathrm{C}$ for $15 \mathrm{sec}, 50^{\circ} \mathrm{C}$ for $10 \mathrm{sec}$ and $60^{\circ} \mathrm{C}$ for $1 \mathrm{~min}$. The mRNA level of each PCR product was estimated by the StepOne software v2.0 and normalized to the GAPDH mRNA level.

Cell culture, transfection and single clone collection. Human cervical squamous cell carcinoma cell line SiHa which was obtained from the American Type Culture Collection was incubated in MEM-n supplemented with $10 \%$ FBS in presence of $5 \% \mathrm{CO}_{2}$. E2-EPF shRNA and control shRNA plasmid vector were purchased from Santa Cruz. Transfection was done when cells were $\sim 70-80 \%$ confluency. All the procedures are followed by the manufacturer's protocol. Forty-eight hours after transfection, cells were selected by puromycin (Santa Cruz, USA) with concentration of $0.5 \mu \mathrm{g} / \mathrm{ml}$ for 2 weeks. The positive cells were harvested and implanted into 96-well plates with single one in each well and changed medium every week. The positive clone were selected and subjected for real-time PCR detection of mRNA and western blot examination of protein expression.

Western blot analysis. Cells were harvested and lysed on ice for $30 \mathrm{~min}$ in M-PER tissue protein extraction reagent (Thermo Scientific, USA) containing complete protease inhibitor mixture (Roche Diagnostics, USA). The lysates were subjected to centrifugation at $14800 \mathrm{~g}$ for $15 \mathrm{~min}$ and the soluble fraction was collected. Protein concentrations were measured using BCA protein assay kit (Thermo Scientific). Equal amounts of protein $(30 \mu \mathrm{g})$ were loaded and probed with anti-E2-EPF antibody C-term (Abgent, USA). The intensity of each band was normalized to the intensity of the $\beta$-actin band. The lysate of HL-60 cells were used as an E2-EPF-positive control.

Luciferase assays. Cells $\left(2 \times 10^{4}\right)$ of E2-EPF knockdown single clone, E2-EPF(-), were seeded at 96-well plate and incubated overnight. For each transfection, $50 \mathrm{ng}$ HIF-1 luciferase reporter vector (Panomics, USA) was mixed in $0.2 \mathrm{ml}$ Opti-MEM (Invitrogen, USA), and a precipitate was formed using Lipofectamine 2000 (Invitrogen) according to the protocol recommendations. After transfection for $24 \mathrm{~h}$, cells were harvested and extracts prepared with Glo Lysis buffer (Promega, USA). Luciferase activity was measured in extracts from triplicate samples using the Bright-Glo Luciferase Assay system (Promega).
Proliferation and invasion assays. Cells $\left(2 \times 10^{3}\right)$ E2-EPF(-), control and normal cells were implanted in triplicate into 96-well plate and allow attaching overnight and the proliferation assay was performed according to the instructions of CellTiter 96 Non-Radioactive Cell Proliferation assay (Promega). Record the absorbance at $570 \mathrm{~nm}$ with 96 -well plate reader.

The invasion assay was done following the manufacturer's protocol (BD Biosciences, UK). In brief, following synchronization by serum starvation for $24 \mathrm{~h}$, cell suspensions in $1 \% \mathrm{FBS}$ MEM-n containing $2.5 \times 10^{4}$ cells was added into the 24 -well BioCoat Matrigel invasion chambers and MEM-n containing $20 \%$ FBS was added into the low chamber. After 24-h incubation, the cells on the lower surface of the membrane are stained with Diff-Quik $^{\mathrm{TM}}$ stain kit. The number of cells was scored visually in 8 random, non-overlapping fields at magnification 10x10 using a light microscope.

Chemotherapeutic drug treatments. Cells $\left(4 \times 10^{4}\right)$ of each well were implanted into 96-well plate in triplicate. Drugs were diluted from distilled water or dimethyl sulfoxide stock solutions into culture medium working solutions and added at the following concentrations (Table I). After $24 \mathrm{~h}$ of drug treatment, survival cells were evaluated by the Cell Proliferation assay (Promega). Drug resistance was represented as calculated using the following formula: (absorbance of treated cells)/(absorbance of untreated cells) x $100 \%$.

Cell cycle analysis. Approximately $2 \times 10^{6} \mathrm{E} 2-\mathrm{EPF}(-)$, control and normal cells were fixed overnight in $1.5 \mathrm{ml}$ of $70 \%$ ethanol. Thereafter, cells were centrifuged, the supernatant discarded, and the cell pellet resuspended and washed twice with PBS. After another centrifugation step, cells were resuspended in $300 \mu \mathrm{l}$ PBS staining solution containing $100 \mu \mathrm{g} / \mathrm{ml}$ propidium iodide (Invitrogen/Molecular Probes) and $100 \mu \mathrm{g} / \mathrm{ml}$ freshly prepared RNase A (Qiagen, USA) and were incubated at room temperature for $30 \mathrm{~min}$. FACS analysis was performed using a FACSCalibur (BD Biosciences) at $488 \mathrm{~nm}$. The data were analyzed using CellQuest Pro and ModFit software.

Tumor cell implantation experiments. In vivo experiments were done in accordance with the guidelines for the Care and Use of Laboratory Animals of China-Japan Friendship Hospital. E2-EPF(-), control and normal cells were s.c. injected into the back of 4 -week-old female nude mice with cells at $2 \times 10^{6}$ per mouse. Tumor growth was monitored every 3 days after 6 weeks of inoculation. Mice were sacrificed at day 90 and tumors were excised and weighted.

Statistical analysis. Data are presented as the mean \pm SD. $\mathrm{p}<0.05$ was considered statistically significant. E2-EPF mRNA expression in cervical tumor and normal tissues and tumor weight in in vivo tumorigenesis were compared using Student's t-test. Luciferase assay, MTT assay, invasion assay and chemotherapeutic sensitivity assay was analysed using ANOVA test.

\section{Results}

E2-EPF $m R N A$ are overexpressed in cervical tumor specimens. E2-EPF was significantly overexpressed in tumor tissues relative to normal tissues (Fig. 1A). The average relative expression level 
Table I. Concentration of different drugs used for chemotherapeutic sensitivity test.

\begin{tabular}{lccccc}
\hline Cisplatin $(\mu \mathrm{M})$ & Doxorubicin $(\mathrm{nM})$ & Paclitaxel $(\mathrm{nM})$ & Doxetaxel $(\mathrm{nM})$ & Topotecan $(\mathrm{nM})$ & Etoposide $(\mu \mathrm{g} / \mathrm{ml})$ \\
\hline 0 & 0 & 0 & 0 & 0 & 0 \\
2.5 & 50 & 10 & 3 & 1 & 1 \\
10 & 200 & 20 & 10 & 2 & 10 \\
40 & 800 & 50 & 20 & 4 & 30 \\
\hline
\end{tabular}

A
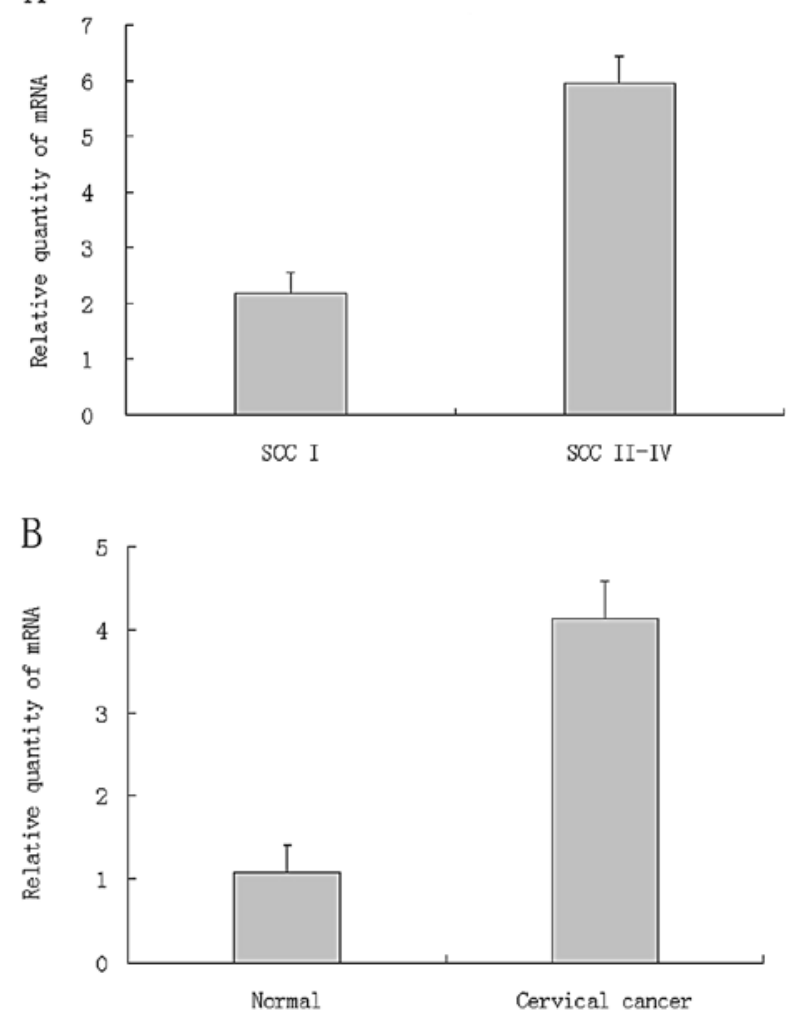

Figure 1. Relative expression levels of E2-EPF were determined by TaqMan real-time PCR. Expression levels were normalized to the housekeeping gene GAPDH. (A) The expression level of E2-EPF is higher in tumor tissues than in normal tissues. (B) The expression level of E2-EPF in tumors at clinical stages II-IV is higher than the tumors at clinical stage I. Each bar indicates the mean $\pm \mathrm{SD}$ of the data collected. $\mathrm{p}<0.05$, tumor vs. normal, I vs II-IV.

in tumor tissues is $\sim 4$-fold of the normal tissues. Relationship between expression level of E2-EPF and clinical stage of the tumor tissues was analyzed. The relative expression level of E2-EPF in tumors at stage I was significantly lower than the tumors at stage II-IV (Fig. 1B).

Establishment of E2-EPF low expression clones. After antibiotics selection, the survival cells were seeded into 96-well plates for E2-EPF(-) single clone selection. All together, $>15$ low expression clones were selected and subjected for real-time PCR examination of mRNA level and western blot detection of protein levels. Fig. 2A shows the relative mRNA level of the representative clones. shRNA decreased the E2-EPF mRNA by more than $80 \%$ in some clones. Fig. $2 \mathrm{~B}$ shows the relative
A

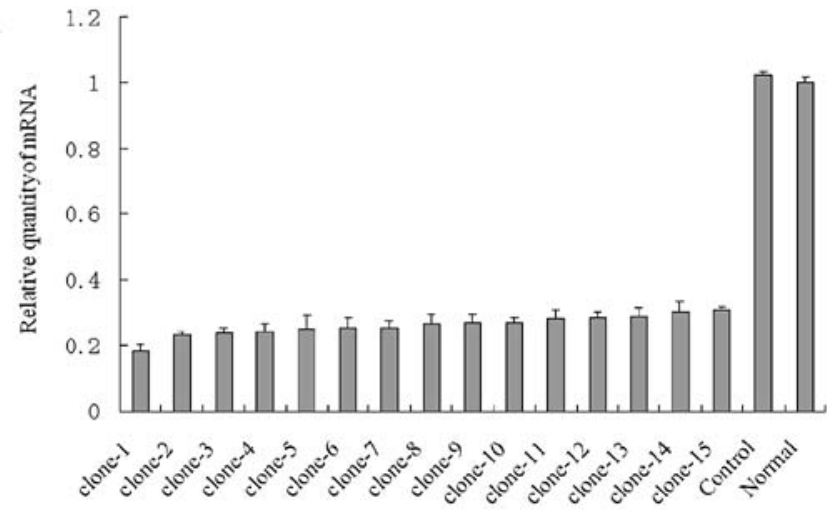

B

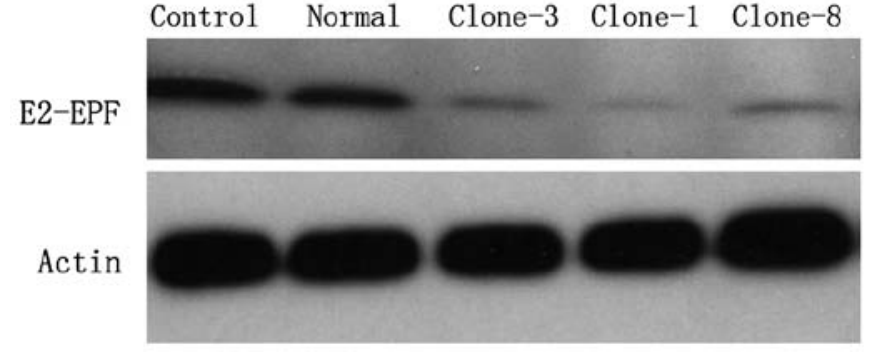

Figure 2. (A) Relative mRNA level of the representative clones detected by TaqMan real-time PCR. (B) Western blot analysis of E2-EPF expression.

protein level. The E2-EPF(-) clones show hardly any protein expression.

Decreased expression of E2-EPF is associated with the lower promoter activity of HIF-1 reporter. Luciferase assay showed that the promoter activity of HIF-1 reporter was significantly decreased in the E2-EPF(-) clone cells. This results indicates that HIF-1 $\alpha$ level may be downregulated in these cells (Fig. 3).

shRNA knockdown decreases growth rate and aggressivity of cells. E2-EPF(-) clone cells displayed a lower proliferative potential compared with normal and control cells (Fig. 4A), indicating that E2-EPF is involved in the growth control of $\mathrm{SiHa}$ cell. The aggressivity of E2-EPF(-) clone cells was significantly decreased (Fig. 4B).

E2-EPF knockdown increased the cells no. in the G0/G1 phase. FACS analysis of propidium iodine-stained cells revealed that E2-EPF knockdown increased the cells no. in the G0/G1 phase. The percentage of cells in G2/M, especially $S$ phase are signifi- 


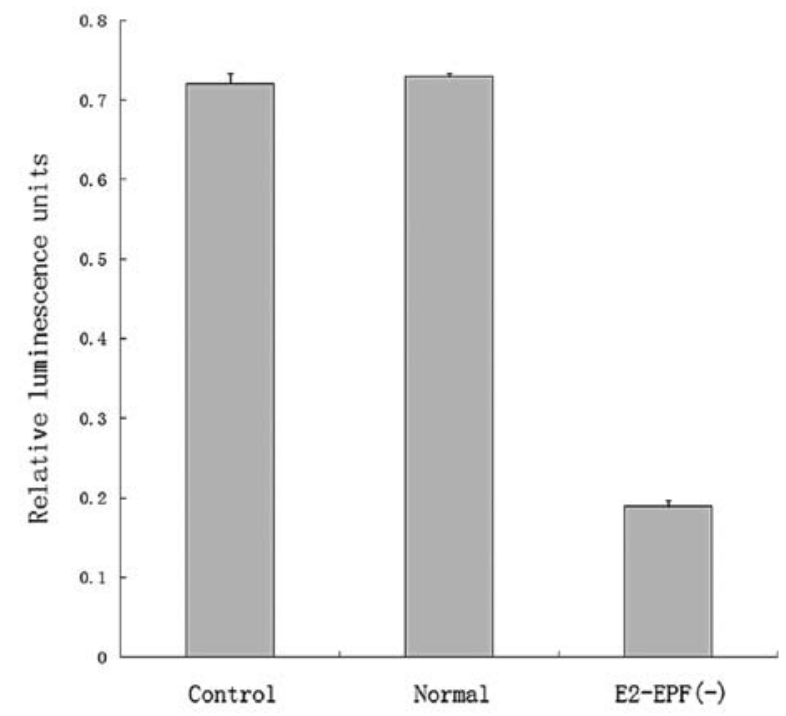

Figure 3. Luciferase activity of control, normal and E2-EPF(-) cells were transfected with the HIF-1 $\alpha$ expression constructs under normoxic condition. Activity was reported as relative luminescence units (RLU). Error bars indicate standard deviations in triplicate assays.

cantly lower in E2-EPF low expression cells than the normal and control cells. The representative results were shown in Fig. 5.

E2-EPF knockdown increased SiHa cell sensitivity to topoisomerase inhibitors. We used different kinds of chemotherapeutic drugs to evaluate the effect of E2-EPF knockdown on cell chemosensitivity, i.e., topotecan, etoposide, doxorubicin, cisplatin, paclitaxel and doxetaxel. E2-EPF knockdown resulted in a significantly greater antiproliferative effect in the topoisomerase I inhibitor (topotecan, Fig. 6A) and II (etoposide and doxorubicin, Fig. 6B and C). In contrast, no significant sensitizing effects of E2-EPF depletion were observed in cisplatin, paclitaxel or doxetaxel (Fig. 6D-F).

E2-EPF knockdown decreases the tumorigenicity of SiHa cell line. To test whether E2-EPF knockdown affects the tumorigenic ability of SiHa cell line or not, certain number of tumor cells were s.c. inoculated into nude mice to monitor the tumor development. As shown in Fig. 7A, weight of tumors from E2-EPF low expression clone cells was $\sim 1 / 4$ of the normal and control cells. Typical tumors are shown in Fig. 7B. These results indicate that E2-EPF may play an important role in tumor development in vivo.

\section{Discussion}

The importance of E2-EPF in human cancers has become evident in recent years, including, breast cancer, colon cancer, and renal cancer (13). To explore the role of E2-EPF in cervical cancer, we examined the expression level of E2-EPF in cervical tumor tissues and normal tissues. Our study demonstrated that the E2-EPF protein was more highly expressed in the cervical tumor tissues than in normal tissues. Also, the expression level was positively correlated with the clinical stage of tumor. These results indicate that E2-EPF high expression may be associated with the tumor growth, cell invasion and/or metastasis. It has
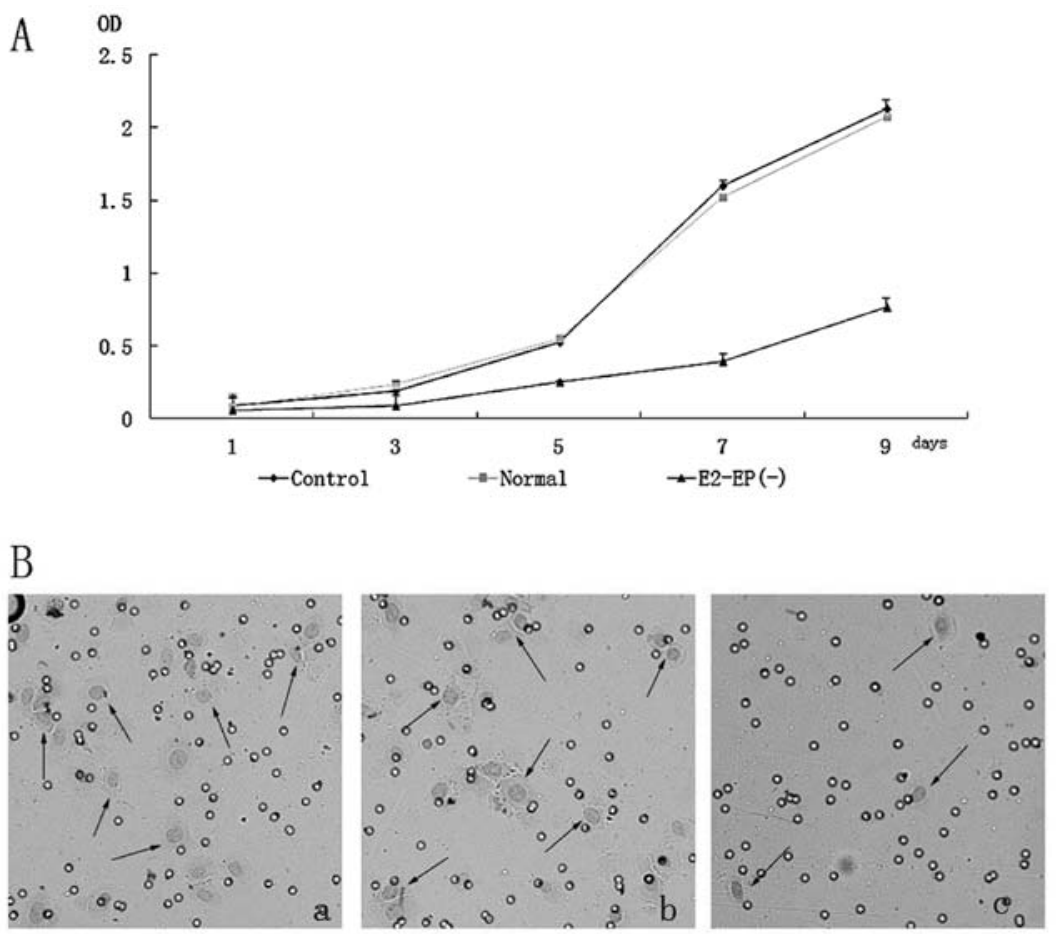

Figure 4. (A) MTT results of the three representative clones. Cells (2x103) E2-EPF(-), control and normal cells were seeded in triplicate into 96-well plate. The relative cell number was determined by the $570 \mathrm{~nm}$ using the MTT assay kit at days 2, 4 and 6. Each point indicates the mean of 3-wells. The data are representative of three independent experiments. shRNA knockdown decreased cell growth, p<0.05, E2-EPF(-) vs normal and control. (B) Cells (2.5x10 $\left.{ }^{4}\right)$ of E2-EPF(-), control and normal cells were seeded in triplicate into 24-well BioCoat Matrigel invasion chamber. Eight fields were selected randomly and counted invasived cells' number as the final results. a, b and c represent control, normal and E2-EPF(-) clone cells respectively. Arrowhead points the cells that migrates the well. Magnification 10x10. 
A
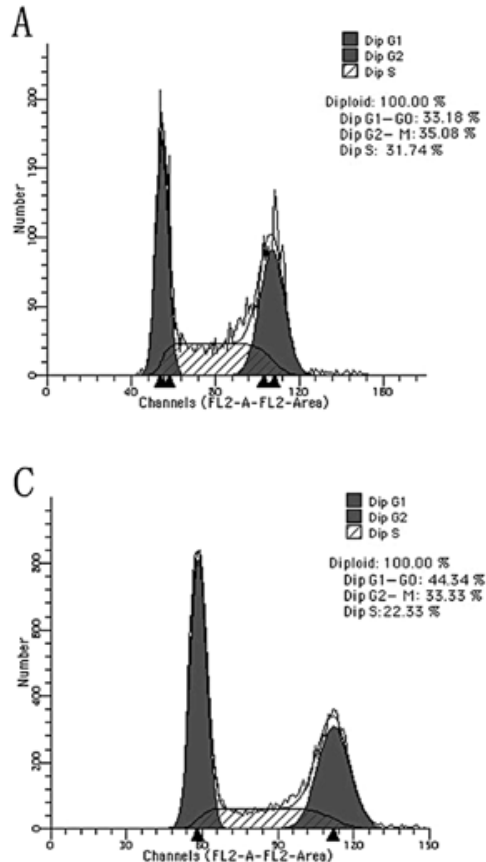

B

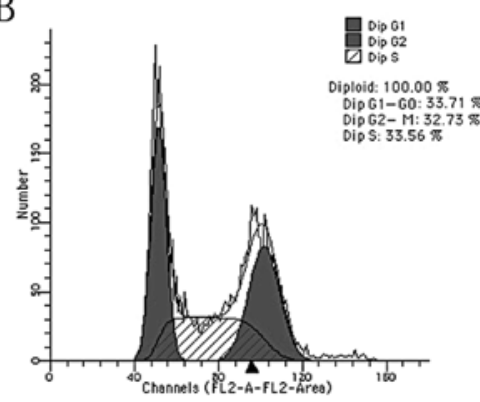

D

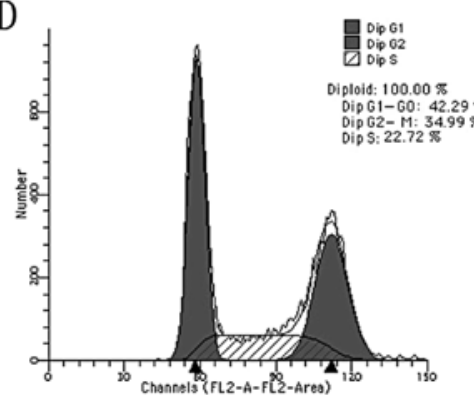

Figure 5. Cell cycle was analysed by flow cytometry. Two clones of E2-EPF(-) (C and D) cells showed a marked decrease in the percentage of $\mathrm{S}$ phase and increased in the percentage of G0/G1 phase than the normal (A) and control (B). Typical results of multiple repeat analysis are shown.

A

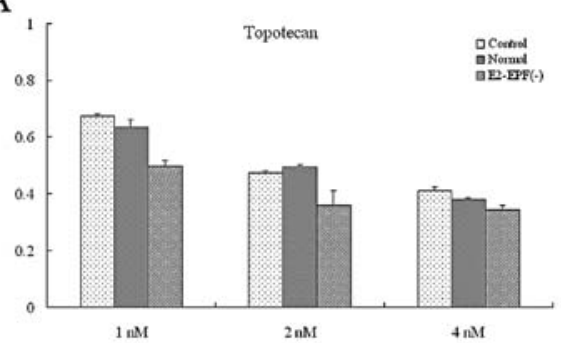

C

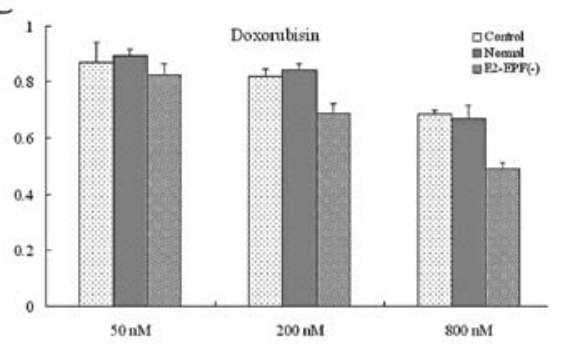

E

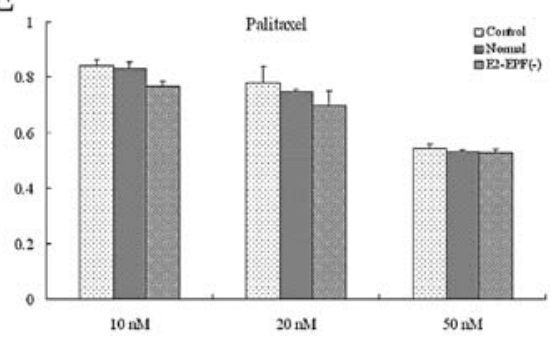

B

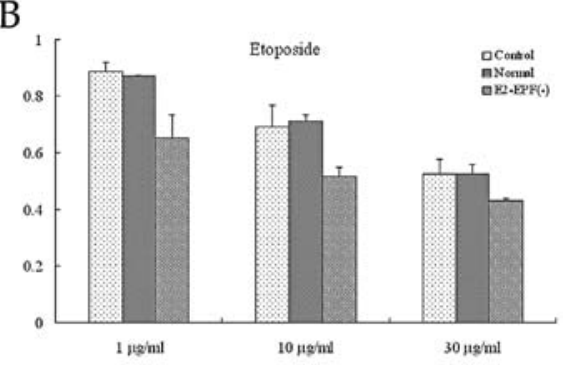

$\mathrm{D}$

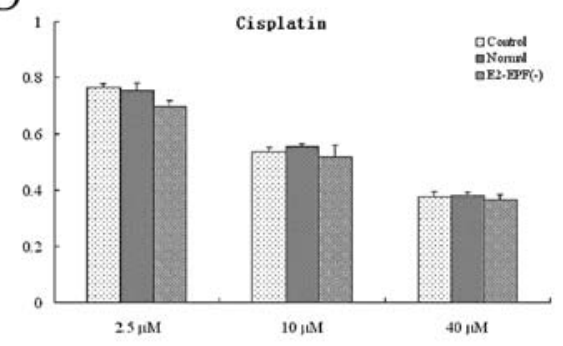

F

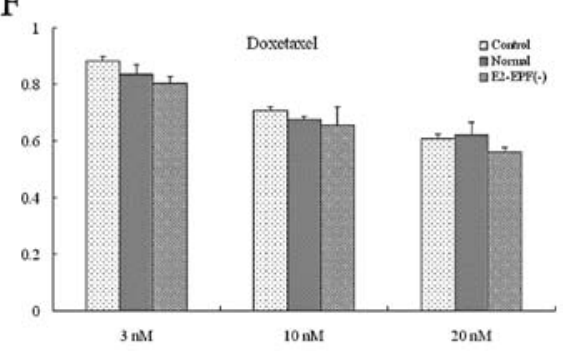

Figure 6. (A) Topotecan (1 or $2 \mathrm{~nm}$ ) was added to treat the control, normal and E2-EPF(-) cells. The relative survival cell no. in E2-EPF(-) cell was significantly lower than the control and normal cells, $\mathrm{p}<0.05$. But no difference was observed in the drug concentration of $4 \mathrm{~nm}$. Among the three groups, $\mathrm{p}>0.05$. (B) Etoposide $(1,10$ and $30 \mu \mathrm{g} / \mathrm{ml})$ was added to treat the three groups. The relative survival cell no. in E2-EPF(-) cell was significantly lower than the control and normal cells, $\mathrm{p}<0.05$. (C) The relative survival cell no. in E2-EPF(-) cell was significantly lower than the control and normal cells, in 200 and $800 \mathrm{~nm}$ Doxorubicin, $\mathrm{p}<0.05$, but no difference was observed in the drug concentration of $50 \mathrm{~nm}, \mathrm{p}>0.05$. (D) Cisplatin $(2.5,10$ and $40 \mu \mathrm{M})$ was added to treat the three groups. There is no significant difference of the relative survival cell no. among the three groups, $\mathrm{p}>0.05$. (E) Paclitaxel (10, 20 and $50 \mathrm{~nm})$ was added to treat the three groups. There is no significant difference of the relative survival cell no. among the three groups, $\mathrm{p}>0.05$. (F) Doxetaxel $(3,10$ and $20 \mathrm{~nm})$ was added to treat the $\mathrm{c}$ three groups. There is no significant difference of the relative survival cell no. among the three groups, $\mathrm{p}>0.05$. 


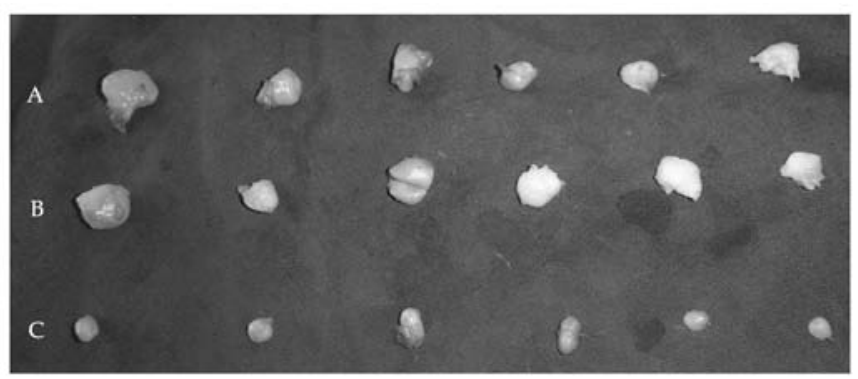

Figure 7. Weight of tumor from the normal cells (A), the control cells (B) and the E2-EPF(-) cells (C) were $2.062 \pm 0.338$ and $0.593 \pm 0.339$ g respectively.

been showed that E2-EPF was significantly associated with poor prognosis of esophageal cancer (11). The role of E2-EPF in cervical cancer prognosis is known and more cases of clinical specimens need to be collected and analysed.

To further demonstrate the role of E2-EPF in cervical cancer, we downregulated its expression with a silencing shRNA plasmid vector. Cell growth and invasion were examined by MTT assay and invasion assay, respectively. Interestingly, downregulation of E2-EPF decreased the cell growth rate and aggressivity which indicates that E2-EPF may play an important role in the cell growth and invasion. This was further approved by cell cycle analysis. In vivo study showed that the tumorigenicity of the E2-EPF knockdown cell decreased dramatically. All these results indicate that E2-EPF may be one of the key factors which is involved in cell growth, tumor development. Targeting E2-EPF pathway may be a potential therapeutic method of cervical cancer treatment. Using bortezomib, a proteasome inhibitor, can decrease the function HIF-1-VEGF pathway in SiHa tumor cell line (14). As previous study showed that E2-EPF increased the expression of HIF-1 $\alpha$ through degrading the tumor suppressor pVHL (7). It is quite promising to find new E2-EPF inhibitors which can be developed into new anti-cancer drugs.

FACS analysis revealed a marked increase in the percentage of the G0/G1 phase cells and a obvious decrease of the $S$ phase after E2-EPF knockdown. It is reported that the anaphase-promoting complex (APC) is an E3 ubiquitin ligase that regulates mitosis and $\mathrm{G} 1$ by sequentially targeting cell cycle regulators for ubiquitination and proteasomal degradation. E2-EPF is a critical, unique component of the APC ubiquitination pathway (15). E2-EPF acts as an APC/C auxiliary factor that promotes mitotic exit (16). Our results showed that decreased E2-EPF expression leading to the decreased cell number, mainly in the $S$ phase, which may be due to the function of E2-EPF in the APC complex. Previous study using HeLa, the cervical adenocarcinoma cell line, found that E2-EPF mRNA expression correlated with genes involved in mitotic surveillance, but RNAi mediated knockdown of the E2-EPF protein did not alter cell cycle distribution or affect the proliferation (10). Our study showed a quite different result using $\mathrm{SiHa}$, the cervical squamous cancer cell line. Therefore, we supposed that the role of E2-EPF in cell growth and cell cycle is different depending on the cell type. Evaluation of additional cell lines representative of other cervical cancer cell lines for effects of E2-EPF depletion may therefore be warranted.

Studies showed that E2-EPF ubiquitin carrier protein associates with and targets pVHL for ubiquitin-mediated proteolysis in cells, thereby stabilizing HIF-1 $\alpha$ (7). The heterodimeric transcription factors HIF-1 and HIF-2, composed of HIF-1 $\alpha$ or HIF-2 $\alpha$ and HIF-1 $\beta$, increase expression of a number of hypoxia-inducible genes including the gene encoding vascular endothelial growth factor (VEGF), which promotes tumor growth and vascularization $(7,17)$. Interestingly, recent study using the sporadic papillary renal cell carcinoma found that multiple hypoxia-responsive elements within the E2-EPF promoter, which demonstrated that E2-EPF is a hypoxia inducible gene directly regulated via HIF-1 (13). Our study also demonstrated that HIF-1 $\alpha$ expression was decreased in the E2-EPF(-) clone cells, which indicate that E2-EPF may also play an important role in cervical cancer invasion and metastasis through effects of the pVHL-HIF pathway.

Here we found that E2-EPF knockdown increased the cell sensitivity to the topoisomerase I inhibitor (topotecan) and II (etoposide and doxorubicin). The possible explanation for the increased antiproliferative effect of Topo II inhibitors following E2-EPF knockdown is that Topo II protein levels were increased. Alternatively, E2-EPF may decrease drug sensitivity by involving in the turnover of Topo II inhibitor-induced Topo II-DNA complexes, thereby enabling repair of DNA damage (10). The mechanism of E2-EPF knockdown on the increased sensitivity to the topoisomerase I inhibitor is still unknown. These data suggest that combined administration of topoisomerase directed drugs and E2-EPF inhibitors may enhance their clinical effectiveness.

In conclusion, E2-EPF is overexpressed in cervical squamous cancer specimens and its expression level positively correlated with the clinical stage. Downregulation of E2-EPF by RNAi results in a decreased expression level of HIF-1 $\alpha$. E2-EPF knockdown decreases cell growth, cell invasion and tumorigenicity of $\mathrm{SiHa}$ cell line and increases the chemosensitivity to topoisomerase inhibitors. E2-EPF plays an important role in the tumorigenesis and development. Targeting of E2-EPF pathway may be a new therapeutic method of cervical tumor treatment.

\section{Acknowledgements}

We thank Chun-h. Xu for excellent technical assistance. This study was partially supported by the Japan-China Sasakawa Medical Fellowship.

\section{References}

1. Brahimi-Horn $\mathrm{C}$ and Pouyssegur $\mathrm{J}$ : When hypoxia signalling meets the ubiquitin-proteasomal pathway, new targets for cancer therapy. Crit Rev Oncol Hematol 53: 115-123, 2005.

2. Liu Z, Diaz LA, Haas AL and Giudice GJ: cDNA cloning of a novel human ubiquitin carrier protein. An antigenic domain specifically recognized by endemic pemphigus foliaceus autoantibodies is encoded in a secondary reading frame of this human epidermal transcript. J Biol Chem 267: 15829-15835, 1992.

3. Liu Z, Haas AL, Diaz LA, Conrad CA and Giudice GJ: Characterization of a novel keratinocyte ubiquitin carrier protein. J Biol Chem 271: 2817-2822, 1996.

4. Welsh JB, Zarrinkar PP, Sapinoso LM, et al: Analysis of gene expression profiles in normal and neoplastic ovarian tissue samples identifies candidate molecular markers of epithelial ovarian cancer. Proc Natl Acad Sci USA 98: 1176-1181, 2001.

5. Wagner KW, Sapinoso LM, El-Rifai W, et al: Overexpression, genomic amplification and therapeutic potential of inhibiting the UbcH10 ubiquitin conjugase in human carcinomas of diverse anatomic origin. Oncogene 23: 6621-6629, 2004. 
6. Rhodes DR, Yu J, Shanker K, et al: Large-scale meta-analysis of cancer microarray data identifies common transcriptional profiles of neoplastic transformation and progression. Proc Natl Acad Sci USA 101: 9309-9314, 2004.

7. Jung CR, Hwang KS, Yoo J, et al: E2-EPF UCP targets pVHL for degradation and associates with tumor growth and metastasis. Nat Med 12: 809-816, 2006.

8. Harada H, Kizaka-Kondoh S, Li G, et al: Significance of HIF-1active cells in angiogenesis and radioresistance. Oncogene 26 : 7508-7516, 2007.

9. Ohh M: pVHL's kryptonite: E2-EPF UCP. Cancer Cell 10: 95-97, 2006.

10. Tedesco D, Zhang J, Trinh L, et al: The ubiquitin-conjugating enzyme E2-EPF is overexpressed in primary breast cancer and modulates sensitivity to topoisomerase II inhibition. Neoplasia 9: 601-613, 2007.

11. Chen MF, Lee KD, Lu MS, et al: The predictive role of E2-EPF ubiquitin carrier protein in esophageal squamous cell carcinoma. J Mol Med 87: 307-320, 2009.
12. Lim JH, Jung CR, Lee CH and Im DS: Egr-1 and serum response factor are involved in growth factors- and serum-mediated induction of E2-EPF UCP expression that regulates the VHL-HIF pathway. J Cell Biochem 105: 1117-1127, 2008.

13. Roos FC, Evans AJ, Brenner W, et al: Deregulation of E2-EPF ubiquitin carrier protein in papillary renal cell carcinoma. Am J Pathol 178: 853-860, 2010.

14. Birle DC and Hedley DW: Suppression of the hypoxia-inducible factor-1 response in cervical carcinoma xenografts by proteasome inhibitors. Cancer Res 67: 1735-1743, 2007.

15. Wu T, Merbl Y, Huo Y, Gallop JL, Tzur A and Kirschner MW: UBE2S drives elongation of K11-linked ubiquitin chains by the anaphase-promoting complex. Proc Natl Acad Sci USA 107: 1355-1360, 2010.

16. Garnett MJ, Mansfeld J, Godwin C, et al: UBE2S elongates ubiquitin chains on APC/C substrates to promote mitotic exit. Nat Cell Biol 11: 1363-1369, 2009.

17. Pugh CW and Ratcliffe PJ: Regulation of angiogenesis by hypoxia: role of the HIF system. Nat Med 9: 677-684, 2003. 